\title{
Plastic, fantastic! Phenotypic variance in the transcriptional landscape of the grape berry
}

\author{
Alisdair R Fernie* and Takayuki Tohge \\ Please see related Research: http://genomebiology.com/2013/14/6/R54
}

\begin{abstract}
Evaluation of the common grapevine Vitis vinifera across multiple harvests and field locations provides important insights into crop transcriptional plasticity under diverse agricultural regimes.
\end{abstract}

Phenotypic plasticity is a cross-kingdom phenomenon whereby a single genotype is capable of producing a range of phenotypes as a function of its environment, conferring a fitness advantage to the individual when challenged by changing environments [1]. Although this phenomenon is evident in a broad range of species and is of increasing importance to agriculture given the current climatic instability [2], our understanding of the genetic and molecular mechanisms underlying phenotypic plasticity and its counterpoint, environmental canalization [3], remains rather limited. This is despite the fact that the balance between plasticity and robustness, in terms of the ability to respond rapidly to environmental pressure without the need for natural selection and the buffering of phenotypes against environmental variation, is generally regarded to be crucial for optimal fitness $[4,5]$. In their article in this special issue on plant genomics, Dal Santo et al. describe an approach to understand phenotypic plasticity at the transcript level in clones of grape grown in 11 different locations and on differing rootstocks across three independent stages of berry development [6]. Specifically, they harvested homogeneously and uniformly ripened fruit from harvests of Vitis vinifera cultivar Corvina at 11 open field sites in the Verona area of Italy over three consecutive years and subjected these to microarray analyses (Figure 1). For this purpose, the NimbleGen 090918_Vitus_exp_HX12 microarray, affording coverage of over $98 \%$ of the genes

*Correspondence: fernie@mpimp-golm.mpg.de

Max-Planck-Institute of Molecular Plant Physiology, Am Mühlenberg 1, 14476 Potsdam-Golm, Germany encoded in the grape genome $[7,8]$, was used to evaluate transcript levels in grapes of three different developmental stages, as assessed by traditional ripening metrics; metabolic biomarkers were also used. As such, this experiment likely represents one of the broadest in field transcriptomics experiments published to date and one from which many important fundamental and applied conclusions can be drawn.

\section{The broader significance of phenotypic plasticity}

Current cultivation practice in vineyards, whereby clonally propagated scions (shoots used for grafting) are grafted onto disease-resistant rootstocks of a different cultivar, renders the grapevine an interesting crop in which to study phenotypic plasticity, given that phenotypic variance driven by genetic factors is very much minimized (although not entirely removed due to genetic differences in rootstock). Indeed, many other crop plants are vegetatively propagated, including many fruit tree species, as well as vegetable crops propagated by stolons, runners, bulbs, tubers or corms. Understanding phenotypic plasticity is also of interest in species with more varied genetics since it will undoubtedly provide critical information to guide next-generation crop improvement strategies.

Previous studies examining transcriptional responses to environmental changes have been performed in model organisms such as Drosophila, nematode and mouse, as well as more exotic species such as marine coral, sparrow and salmon. The most recent and most extensive of these studies was carried out by Zhou et al. in Drosophila, revealing that the majority of the transcriptome shows robust expression across a diverse range of environmental challenges, with only approximately $15 \%$ of transcripts being plastic [5]. The plastic transcripts were split into those with high or low genetic variability. They did, however, include a large number of modules of genes, including transcription factors and non-coding RNAs, presumed to play a role in chromatin structure, as well as a range of glucosyltransferases and cytochrome P450s involved in xenobiotic metabolism. Furthermore, analysis of the dataset was compared with the prevailing models 


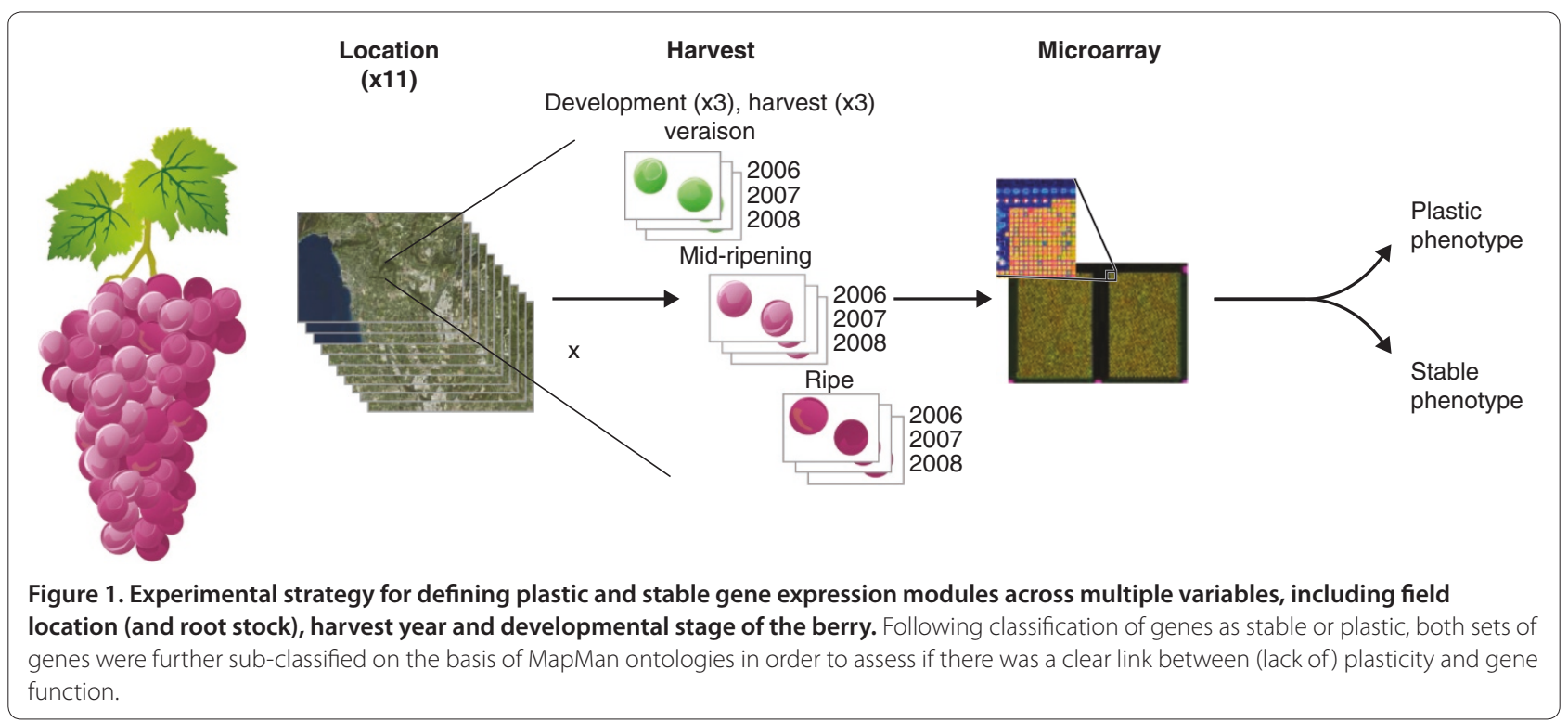

of the genetic basis of phenotypic plasticity. Under the allelic sensitivity model, allele type affects the mean value of a phenotype, whereas under the gene regulation model, plasticity is a trait in itself, which is under the control of regulatory loci [9]. Tellingly, the data of Zhou et al. support the gene regulatory model, as do many other recent studies [9].

\section{Plasticity in the grapevine berry}

Dal Santo et al. appraised the level of plasticity in transcript levels in connection with varying the environment, either with regard to field location (and in some instances rootstock used) or with regard to the cultivation year. As stated earlier, the common grapevine is clonally propagated and thus studies in this species focus on gene regulatory plasticity. Phenotypic plasticity in crop species can be costly; in grapes, the same clone has been documented to show variability within individual berries, among berries in a cluster, between clusters on a vine and among vines in the vineyard. Thus, berry quality may be affected in an unpredictable manner and developmental factors such as uniformity of ripening may be compromised.

In analyzing the berry transcriptome at the scale they did, Dal Santo et al. were able to identify transcripts expressed under normal and unusual weather conditions in addition to defining the plastic complement of the grape transcriptome. They achieved this by means of a carefully planned and executed study in which the 11 vineyards chosen maximized differences in both environmental conditions and agricultural practices across the Verona region. Each sample was taken in triplicate on the same day and spanned three developmental stages (Figure 1), thus allowing multiple statistical comparisons to be made. Firstly, cluster analysis of grape berries across multiple years, but from a subset of the vineyards, revealed a number of interesting traits. Namely, analysis of veraison, the onset of ripening, revealed sets of genes related to: (1) DNA/RNA metabolic processes and transcription factors; (2) hormone metabolism and redox balance; (3) cell wall expansion, hemicellulose modification and carbohydrate dissimilation; and (4) disease resistance and heat shock factors. Mid- and late-ripening stages were instead characterized by major differences in transcripts associated with secondary metabolites.

The data from later harvest points were supported by comparative metabolome analyses between the harvests, which additionally indicated complementary variance in stilbene compounds. This important finding highlights that the massive reprogramming of the berry transcriptome in response to the environment includes metabolic pathways underlying quality traits that are essential to the commercial value of the crop. A second comparison involving data of a single harvest from all 11 vineyards revealed that the proportion of genes that were plastic in the grape berry was of a similar magnitude to that previously reported for Drosophila [5]. Interestingly, there was considerable overlap between the functional classifications of both grape and Drosophila lists, with 'translation', 'nucleobase, nucleoside, nucleotide and nucleic acid metabolic processes', 'regulation of gene expression, epigenetic', 'transcription factor activity' and the glutathione $S$-transferase family being highly represented in the plastic genes of both species. Unsurprisingly, quite distinctive gene functions are also reported for the grape gene set, including several homologs of genes involved in floral transition and organ identify, such as EARLY FLOWERING, CONSTANS, 
FRIGIDA and SEPALLATA being reported as plastic. Remarkably, the combined expression level signature of these plastic transcripts was demonstrated to be a powerful biomarker largely able to separate berries on the basis of the vineyard in which they were grown irrespective of their developmental stage. Further studies aimed at ascertaining whether specific transcript changes were associated with groups of vineyards sharing either common agricultural practices or environmental features. Importantly, only the trellising system and the geographical area could be statistically linked to these changes, and the contribution of the four different rootstock genotypes to the plastic expression of berries is only marginal. This is of particular importance when the data are compared with the models of plasticity mentioned earlier, since it indicates that the plastic genes here are exclusively driven by gene regulatory mechanisms, and not allelic differences. In addition, the proportion of plastic genes reported here being similar to those in the more highly genetically diverse Drosophila population, in combination with the nature of the identified genes displaying plasticity, together provide strong evidence in support of the gene regulatory model of plasticity.

\section{Concluding remarks}

As well as providing important input into the debate on the causes of plasticity, these data are likely to be highly important with regard to crop improvement. The data indicate that veraison is a critical period at which the seasonal climate exerts highest effect, whereas microenvironment and agronomic practices are of only marginal importance, with oxidative stress being of particular note at this developmental stage. During later stages of development, the influence of climate wanes and, accordingly, those of the micro-environment and agronomic practices come into their own, with the exception of transcripts associated with secondary metabolism and indeed the metabolites themselves. Many of the plastic genes identified both at veraison and during berry development are likely well worth looking at in the context of the regulation of ripening and, as such, may represent important targets for crop improvement. The recent sequencing of tomato methylome [10] and the suggested role of epigenetic factors in the ripening process further underscore this statement. As Dal Santo et al. themselves acknowledge, the causality within such associations will certainly require further investigation. Although to date traditional breeding has led to major advances in agriculture, including the green revolution, it remains a relatively inefficient process with an enormous opportunity for improvement. Given that many studies highlight the difficulties in transposing lab results into the field, the need for large-scale in-field testing (of the scale performed by Dal Santo et al.) is great. Their study represents an important benchmark for post-genomics era crop genetics, not only in shedding new light on the decade old questions of mechanisms underlying phenotypic plasticity and canalization, but also for identifying new tools for trait identification and crop improvement.

\section{Competing interests}

The authors declare they have no competing interests.

\section{Acknowledgements}

Work in the Fernie laboratory focused on phenotypic plasticity is funded in the framework of Deutsche Israeli Projekt FE 552/12-1, which is administered by the Deutsche Forschungsgemeinschaft.

Published: 7 June 2013

\section{References}

1. Bradshaw AD, Caspari EW, Thoday JM: Evolutionary significance of phenotypic plasticity in plants. Adv Genet 1965, 13:115-155.

2. Bradshaw AD, Hardwick K: Evolution and stress - genotypic and phenotypic components. Biol J Linn Soc Lond 1989, 37:137-155.

3. Waddington $\mathrm{CH}$ : Canalization of development and the inheritance of acquired characters. Nature 1942, 150:563-565.

4. Gibson G: Decanalization and the origin of complex disease. Nat Rev Genet 2009, 10:134-140.

5. Zhou S, Campbell TG, Stone EA, Mackay TF, Anholt RR: Phenotypic plasticity of the Drosophila transcriptome. PLoS Genet 2012, 8:e1002593.

6. Dal Santo S, Tornielli GB, Zenoni S, Fasoli M, Farina L, Anesi A, Guzzo F, Delledonne M, Pezzotti M: The plasticity of the grapevine berry transcriptome. Genome Biol 2013, 14:R54.

7. Jaillon O, Aury JM, Noel B, Policriti A, Clepet C, Casagrande A, Choisne N, Aubourg S, Vitulo N, Jubin C, Vezzi A, Legeai F, Huqueney P, Dasilva C, Horner D, Mica E, Jublot D, Poulain J, Bruyère C, Billault A, Segurens B, Gouyvenoux M, Ugarte E, Cattonaro F, Anthouard V, Vico V, Del Fabbro C, Alaux M, Di Gaspero $G$, Dumas $V$, et al:: The grapevine genome suggests ancestral hexaploidization in major angiosperm phyla. Nature 2007, 449:463-467.

8. Fasoli M, Dal Santo S, Zenoni S, Tornielli GB, Farina L, Zamboni A, Porceddu A, Venturini L, Bicego M, Murino V, Ferrarini A, Delledonne M, Pezzotti M: The grapevine expression atlas reveals a deep transcriptome shift driving the entire plant into a maturation program. Plant Cell 2012, 24:3489-3505.

9. Via S, Gomulkiewicz R, De Jong G, Scheiner SM, Schlichting CD, Vantienderen $\mathrm{PH}$ : Adaptive phenotypic plasticity: consensus and controversy. Trends Ecol Evol 1995, 10:212-217.

10. Zhong S, Fei Z, Chen YR, Zheng Y, Huang M, Vrebalov J, McQuinn R, Gapper N, Liu B, Xiang J, Shao Y, Giovannoni JJ: Single-base resolution methylomes of tomato fruit development reveal epigenome modifications associated with ripening. Nat Biotechnol 2013, 31:154-159.

doi:10.1186/gb-2013-14-6-119

Cite this article as: Fernie AR, Tohge T: Plastic, fantastic! Phenotypic variance in the transcriptional landscape of the grape berry. Genome Biology 2013, 14:119. 